\title{
Survival differences of patients with ureteral versus pelvicalyceal tumours: a systematic review and meta-analysis
}

\author{
Krystian Kaczmarek, Artur Lemiński, Adam Gołąb, Marcin Słojewski
}

\begin{abstract}
Department of Urology and Urological Oncology, Pomeranian Medical University, Szczecin, Poland
\end{abstract}

Submitted: 16 February 2018; Accepted: 11 April 2018;

Online publication: 21 November 2019

Arch Med Sci 2021; 17 (3): 603-612

DOI: https://doi.org/10.5114/aoms.2019.89893

Copyright $\odot 2019$ Termedia \& Banach

\section{Abstract}

Introduction: Worse survival in upper tract urothelial carcinoma (UTUC) in the presence of a tumour in the ureter vs. pelvicalyceal tumours is reported in some studies; however, the definition of ureteral involvement (UI) varies across studies. We systematically evaluated evidence regarding the prognostic role of UI in overall and cancer-specific survival of patients with UTUC. Material and methods: A systematic search of PubMed, Scopus and Web of Knowledge was performed in March 2018.

Results: The results were presented as a comparison between ureteral vs. pelvicalyceal tumours. A total of 14,895 patients were identified. Cumulative analyses indicated that $\mathrm{UI}$ worsens cancer-specific survival (CSS) and overall survival (OS), with a pooled HR of $1.52(p<0.001)$ and $1.39(p=0.004)$, respectively.

Conclusions: Our meta-analysis identified UI in UTUC as an adverse prognostic factor.

Key words: nephroureterectomy, prognosis, survival, ureter, transitional cell carcinoma.

\section{Introduction}

Upper tract urothelial carcinoma (UTUC) is an uncommon disease that accounts for only $5 \%$ of all urothelial tumours and $10 \%$ of all renal tumours [1, 2]. It can extend from the pyelocaliceal cavities to the ureteral orifice. Unifocal, small, low-grade tumours with papillary growth and no invasive potential can be treated with kidney-sparing surgery; however, approximately $60 \%$ of patients present with an invasive disease [3]. The gold standard of treatment for patients with an invasive disease is radical nephroureterectomy (RNU) with excision of the bladder cuff. Radical treatment should also be considered in all patients with a normal contralateral kidney when one of the following factors occurs: hydronephrosis, tumour size $>1 \mathrm{~cm}$, high-grade tumour on biopsy, multifocal disease, or previous radical cystectomy for bladder cancer [4].

Clear knowledge on prognostic factors is necessary to identify patients with adverse cancer-related features who may in turn benefit from more aggressive and/or additional treatments. The influence of tumour stage, tumour grade, and lymph node status on prognosis has been well estab-

\author{
Corresponding author: \\ Krystian Kaczmarek \\ Department of Urology \\ and Urological Oncology \\ Pomeranian Medical \\ University \\ 72 Powstańców \\ Wielkopolskich St \\ 70-111 Szczecin, Poland \\ Phone: +4891 4661101 \\ Fax: +48914661100 \\ E-mail: \\ k.kaczmarek.md@gmail.com
}


lished [4-6]. Another potential prognostic variable is the tumour location. According to the guidelines of the European Association of Urology, a tumour within the ureter is a factor of poor prognosis for intravesical recurrence; however, information on its influence on survival after RNU is limited compared with that of other prognostic factors [4]. Most studies that reported tumour location as a significant factor for cancer-specific survival (CSS) or overall survival (OS) were limited by their small sample size, case selection bias, singlecentre nature, or a heterogeneous population $[7,8]$. Furthermore, the definition of ureteral involvement (UI) differs across studies. Consequently, this generates bias in evaluating the association between survival and the presence of tumour within the ureter. Therefore, the goal of this study was to perform a robust evaluation of the prognostic value of UI in UTUC by conducting a systematic review of the literature and a meta-analysis of available data.

\section{Material and methods}

\section{Search strategy}

Two authors (K.K. and A.L.) independently performed an electronic bibliographic search of the Medline, Scopus, and Web of Knowledge databases. The following search keywords were used: "cancer specific survival" or "overall survival" or "survival" and "radical nephroureterectomy" or "upper urinary tract carcinoma" or "transitional cell carcinoma" or "urothelial carcinoma" and "tumor location" or "ureteral involvement" or "prognosis" or "risk factors". Abstract books of major international meetings (European Association of Urology, American Urological Association, European Society of Medical Oncology, and American Society of Clinical Oncology) were hand-searched for potentially relevant studies. Moreover, the references of included studies, and those of a previous systematic review, were checked $[9,10]$. All included studies were published in English. These searches were performed without time restriction. The last search was run on 20 March 2018.

\section{Inclusion criteria}

Based on the Preferred Reporting Items for Systematic Reviews and Meta-analysis (PRISMA) guidelines, the Population, Intervention, Comparator, Outcome, and Study (PICOS) design approach was used to describe study eligibility [11]. Studies were considered relevant to this metaanalysis if they compared patients diagnosed as having UTUC with UI (P) who underwent RNU (I) with those with carcinoma localised only in the renal pelvis and calyces (C) to determine the role of $\mathrm{UI}$ as a predictor of CSS or OS (O) using multi- variable Cox proportional hazards regression analysis (S). Meeting abstracts, case reports, editorials, commentaries, letters, and review articles were excluded.

The following criteria were used to select the studies comparing UI with pelvicalyceal tumours:

- large studies that included more than 100 patients;

- studies that provided a definition of UI;

- studies that excluded patients who underwent contemporary radical cystectomy because of a concomitant muscle-invasive bladder cancer or with history of muscle-invasive bladder cancer;

- studies that provided hazard ratios (HRs) from multivariable Cox proportional hazards regression analysis with their corresponding 95\% confidence intervals (Cls) or studies that provided enough data to calculate Cls.

When more than one study reported results from the same patient cohort, we selected one study with the largest sample size to avoid duplication.

\section{Systematic review process}

After removal of duplications, titles and abstracts of 1250 studies were screened by two authors (K.K. and A.L.) for initial study inclusion [12]. Eighty-five potentially relevant studies were assessed for eligibility based on a full-text evaluation, and 14 studies that met all the inclusion criteria were included in the meta-analysis. The studies included in the analysis were published in 2009-2016. Any disagreements between the two reviewers were resolved by discussion; further disagreements were resolved by consensus with the senior author (M.S.). All authors agreed on the final list of included articles. Moreover, we attempted to contact three authors for additional information; two authors responded by providing additional data $[8,9]$. A PRISMA flow diagram of the study selection is shown in Figure 1.

\section{Quality of data assessment}

All included articles were observational studies and/or retrospective series. The Newcastle-Ottawa Scale, which is an instrument recommended by the Cochrane Collaboration to evaluate the design of nonrandomised studies, was used to assess the quality of the studies. Each study was assessed using the star system, which included three perspectives: selection of the study groups, comparability of the groups, and evaluation of the outcome of interest. Two reviewers (K.K. and A.L.) independently performed the assessment. Quality scores for all studies ranged from 7 to 8 stars (Table I). The trials with seven or more stars were considered to be of sufficient quality for meta-analysis. 


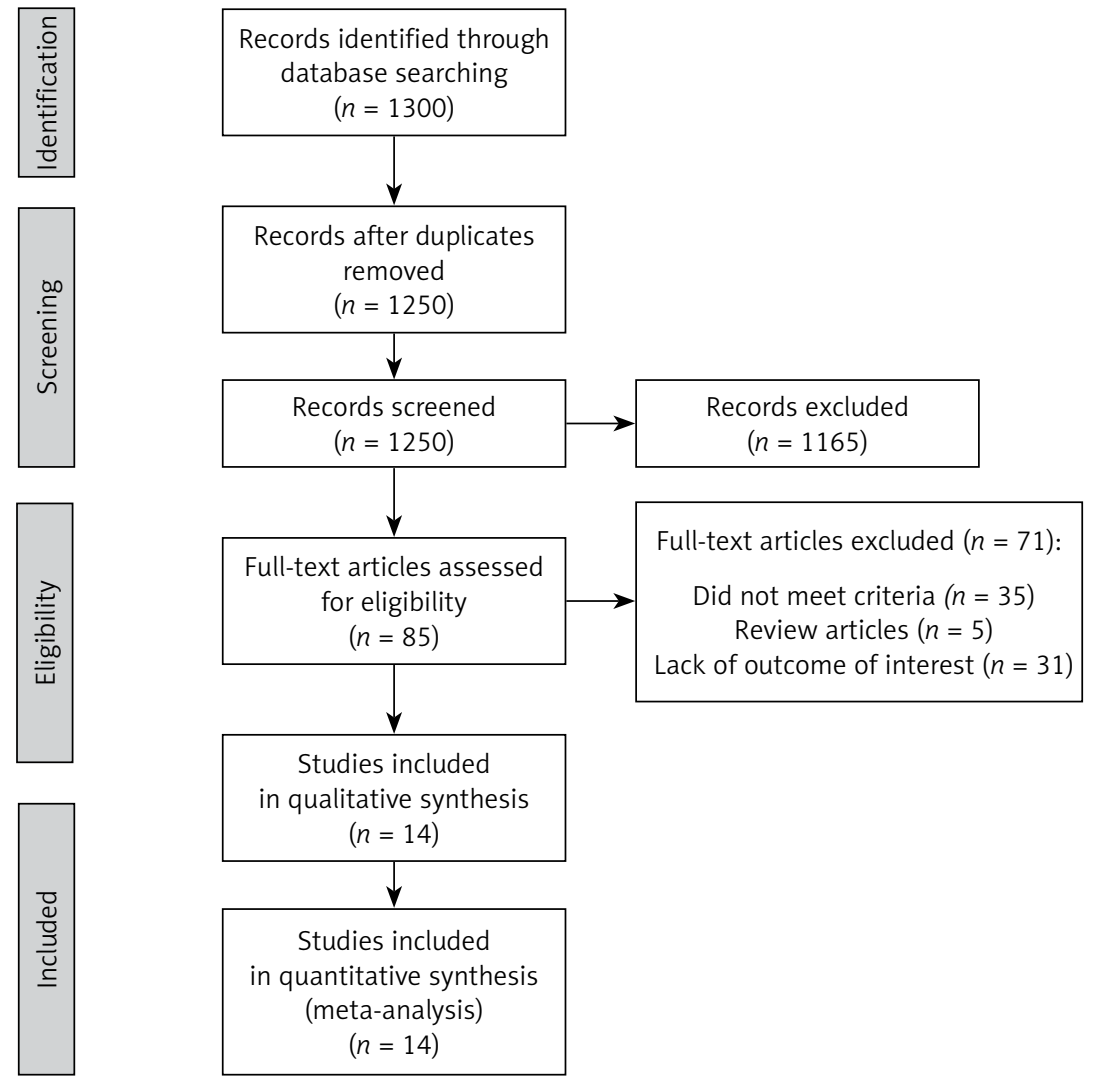

Figure 1. Flowchart showing the process of studies through the selection process

\section{Data extraction}

The following information was extracted from each study: study design, name(s) of author(s), year of publication, recruitment period, number of patients, age, gender, definition of UI, neoadjuvant chemotherapy, and median follow-up period. Subsequently, HRs for UI as a potential predictor of CSS and OS were also obtained. To perform a cumulative analysis, HRs from multivariable Cox proportional hazard regressions analysis with their corresponding 95\% Cls were extracted. Two reviewers (K.K. and A.L) independently performed the data extraction. The other authors (A.G. and M.S.) verified the data.

\section{Outcome measures}

Pooled HRs were used to assess the prognostic role of UI in UTUC. Hazard ratios for CSS and OS were calculated. Patients with and without UI were compared. The outcomes of individual studies were evaluated based on the definition of UI. The following four subgroups were identified:

- tumour present only in the ureter;

- tumour present only in ureter or tumour in the ureter as a dominant lesion in the case of multifocal tumours;

- tumours within two distinct areas (renal pelvis and ureter);
- tumour present only in the ureter and tumours within two distinct areas.

Furthermore, the pooled relative risk (RR) for locally advanced stage UTUC ( $\geq$ pT3) located within the renal pelvis and the ureter was estimated.

\section{Statistical analysis}

The $I^{2}$ statistic was employed to detect heterogeneity across the different studies. The $l^{2}$ statistic describes the percentage of the variability in effect estimates, and a value $>50 \%$ indicated heterogeneity. If no evidence of heterogeneity was found, the fixed-effect inverse variance-weighted method was used to pool the effect size; otherwise, a random effect model was used. Publication bias evaluation was performed with a visual inspection of a funnel plot and using a trim and fill method. Statistical analysis was performed using Review Manager version 5.3 (Cochrane Collaboration, Copenhagen, Denmark) and Comprehensive Meta Analysis version 3 (Biostat, New York, USA).

\section{Results}

The 14 studies in this meta-analysis included 14,895 participants [13-26]. The baseline characteristics of the included studies are summarised in Table I. The number of patients in each selected 


\begin{tabular}{|c|c|c|c|c|c|c|c|c|c|c|c|c|c|c|}
\hline 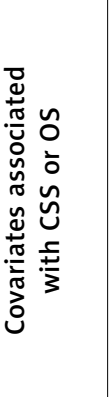 & 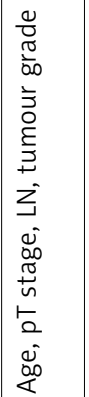 & 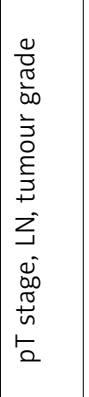 & 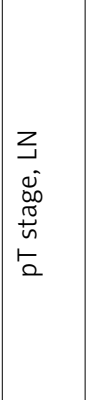 & 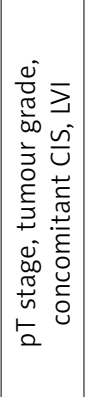 & 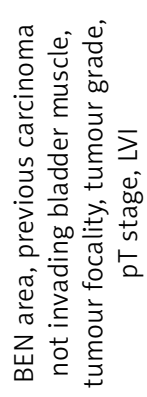 & 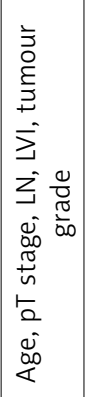 & 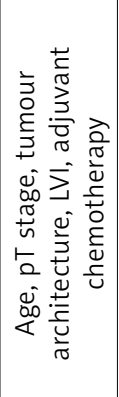 & 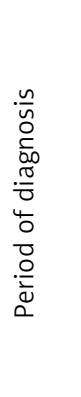 & 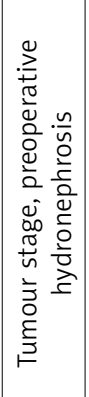 & 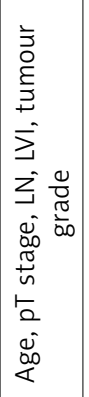 & 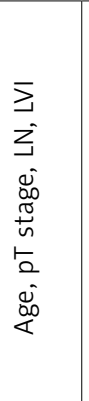 & 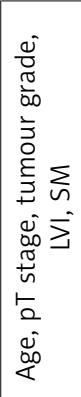 & 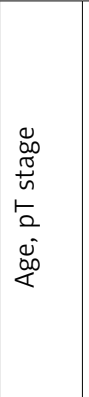 & 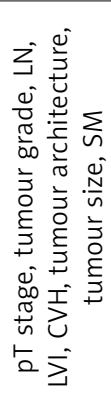 \\
\hline ֶ̃ & $\infty$ & $\infty$ & $\infty$ & $\infty$ & $\lambda$ & $\infty$ & $\infty$ & $\infty$ & $\infty$ & $\wedge$ & $\infty$ & $\infty$ & $\infty$ & $\wedge$ \\
\hline 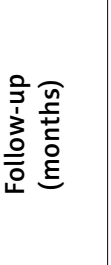 & 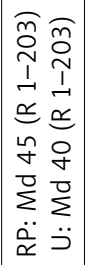 & 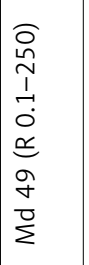 & $\begin{array}{l}\widehat{\alpha} \\
\hat{\alpha} \\
\tilde{N} \\
\sim \\
o \\
o \\
o \\
\infty \\
o \\
\sigma \\
\tilde{\Sigma} \\
\Sigma\end{array}$ & 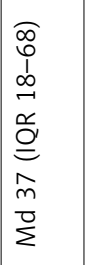 & 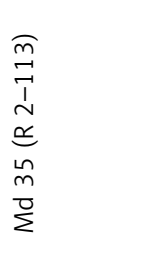 & 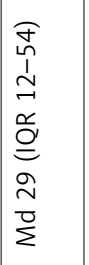 & 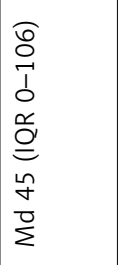 & $\begin{array}{l}0 \\
\dot{0} \\
\infty \\
\sum \\
\sum\end{array}$ & 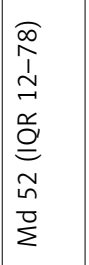 & 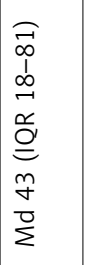 & 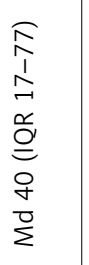 & 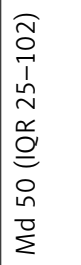 & 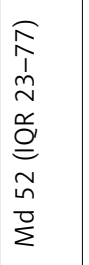 & 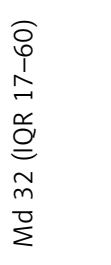 \\
\hline 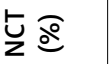 & $\mathbb{z}$ & $\bar{z}$ & 0 & 0 & 0 & 00 & 0 & $\frac{\pi}{z}$ & 0 & 0 & 0 & 0 & 0 & 0 \\
\hline 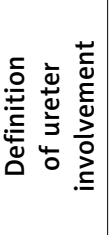 & 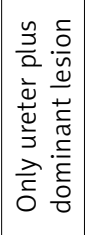 & 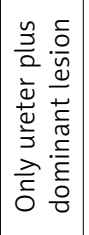 & 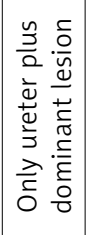 & 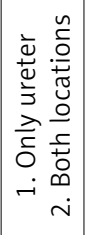 & 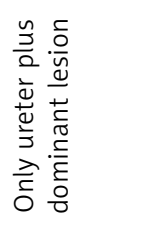 & 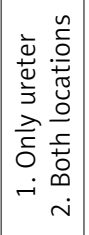 & 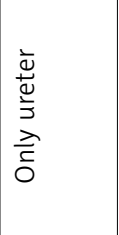 & 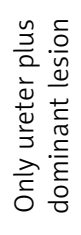 & 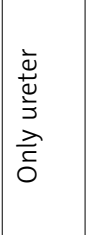 & 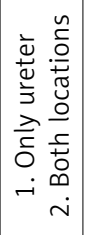 & 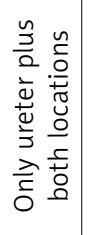 & 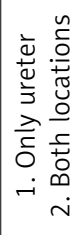 & 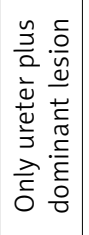 & 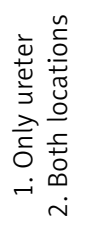 \\
\hline & 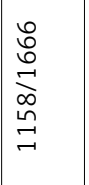 & 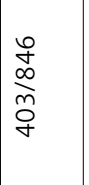 & $\mathbb{\Sigma}$ & 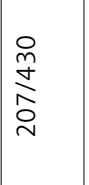 & 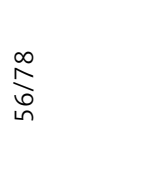 & 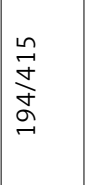 & $\begin{array}{l}\vec{\infty} \\
\stackrel{\infty}{1} \\
\underset{\infty}{-}\end{array}$ & 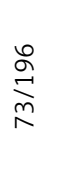 & $\underset{\infty}{\stackrel{P}{\sim}} \underset{\infty}{\stackrel{\infty}{\sim}}$ & 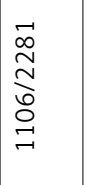 & 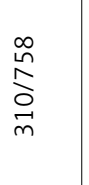 & $\begin{array}{l}\mathcal{N} \\
\stackrel{\sim}{m} \\
\stackrel{N}{O}\end{array}$ & $\underset{\stackrel{\stackrel{q}{\sim}}{\stackrel{f}{\sim}}}{\stackrel{\omega}{\sim}}$ & $\underset{\substack{\tilde{W} \\
m}}{\stackrel{m}{m}}$ \\
\hline 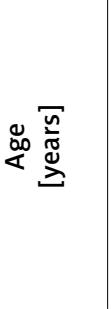 & 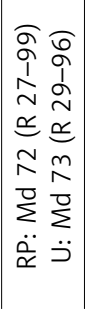 & 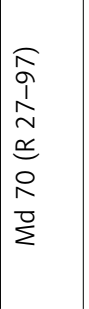 & 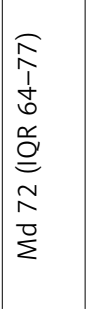 & 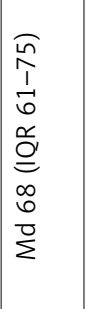 & 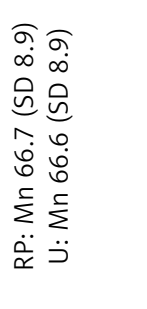 & 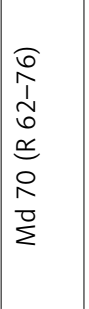 & 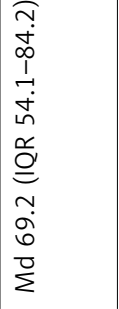 & 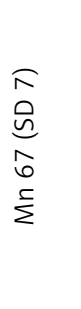 & 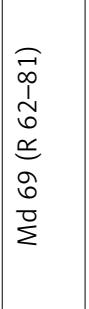 & 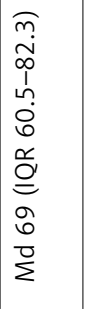 & 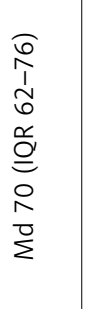 & 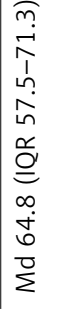 & 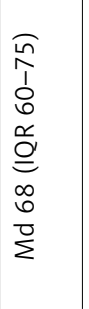 & $\mathbb{z}$ \\
\hline 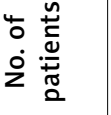 & $\underset{\underset{\sim}{\sim}}{d}$ & 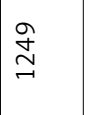 & 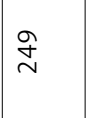 & $\hat{n}$ & $\stackrel{m}{m}$ & ठิ & $\stackrel{\stackrel{0}{\sim}}{\stackrel{\sim}{\sim}}$ & $\stackrel{\leftrightarrow}{\sim}$ & $\vec{\sim}$ & 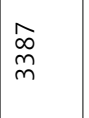 & $\stackrel{\infty}{\stackrel{\circ}{\circ}}$ & 管 & 鬲 & ฉ̊ \\
\hline 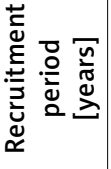 & $\begin{array}{l}1 \\
0 \\
0 \\
N \\
\infty \\
\infty \\
o \\
-1\end{array}$ & 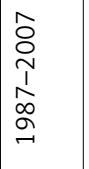 & $\begin{array}{l}\infty \\
o \\
o \\
1 \\
\alpha \\
\alpha \\
\sim\end{array}$ & $\begin{array}{l}0 \\
0 \\
0 \\
i \\
\vdots \\
o \\
\sigma \\
-1\end{array}$ & 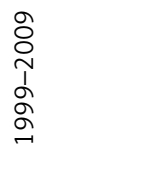 & 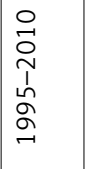 & $\begin{array}{l}\hat{o} \\
0 \\
i \\
1 \\
\infty \\
o \\
-1\end{array}$ & 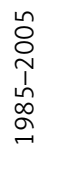 & 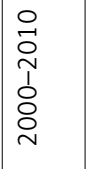 & 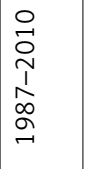 & 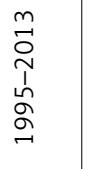 & 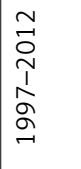 & 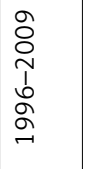 & 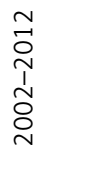 \\
\hline 辛 & $\stackrel{ు}{\unlhd}$ & $\begin{array}{l}\text { 总 } \\
\frac{0}{0}\end{array}$ & 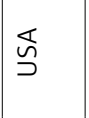 & $\begin{array}{l}0 \\
\stackrel{\circ}{\circ} \\
\end{array}$ & 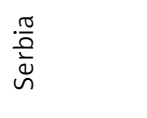 & 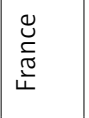 & $\frac{\circ}{\frac{0}{0}}$ & 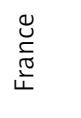 & 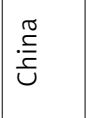 & $\begin{array}{l}\text { 童 } \\
\text { 은 }\end{array}$ & 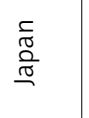 & 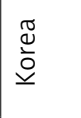 & 旅 & 胥 \\
\hline 害 & 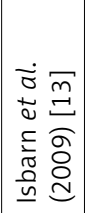 & 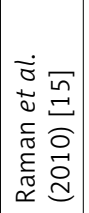 & 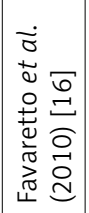 & 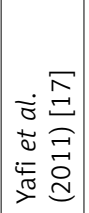 & 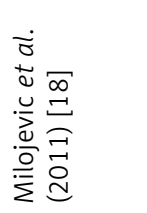 & 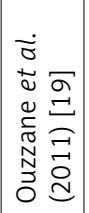 & 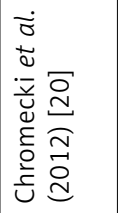 & 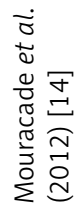 & 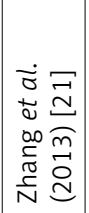 & 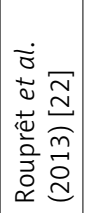 & 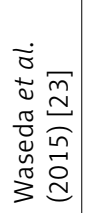 & 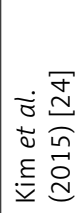 & 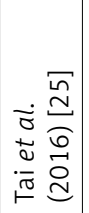 & 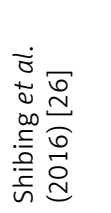 \\
\hline
\end{tabular}


study ranged from 133 to 3387 (mean 1064, median 623). The female to male ratio was approximately $1: 2$. Eight studies analysed UI as a tumour present only in the ureter. In six of those studies $\mathrm{UI}$ was additionally defined as a dominant tumour within the ureter, in the case of multifocal tumours. A dominant lesion was clarified as that with the highest pathologic tumour stage. Five studies analysed two definitions of UI separately: as a tumour located only in the ureter (1) and as a tumour located in two distinct locations (ureter and renal pelvis) (2). Only one included study assessed together a tumour located only in the ureter and tumours in the two distinct locations as UI. The included studies were performed in different geographical areas: four studies were global trials, three studies were conducted in Europe, two studies were conducted in North America, and five studies were conducted in Asia.
Cancer-specific survival after RNU in patients with UI were compared in all studies. A metaanalysis of these studies indicated statistically significant differences in CSS between both groups ( $\mathrm{HR}=1.52,95 \% \mathrm{Cl}: 1.31-1.76 ; p<0.001$; Figure 2). The analysis showed that UI worsened CSS. Moreover, significant heterogeneity was observed across studies $\left(P^{2}=64 \%\right)$. The random effects model was used, and funnel plot inspection revealed presence of publication bias. The trim and fill method suggested that three studies were missing and showed that the HR was 1.39 (95\% Cl: 1.18-1.62).

A subgroup analysis showed no difference in CSS when UI was defined as a tumour located only in the ureter or as a dominant lesion in the ureter (subgroup 2; $\mathrm{HR}=1.11$, 95\% Cl: 0.931.32; $p<0.26)$. Heterogeneity was not observed $\left(I^{2}=0 \%\right)$. Analysis with other subgroups empha-

\begin{tabular}{|c|c|c|c|c|c|c|c|c|}
\hline \multirow{2}{*}{$\frac{\text { Study or subgroup }}{1.1 .1 . \text { Only ureter }}$} & \multirow[t]{2}{*}{$\begin{array}{c}\text { Log } \\
\text { [hazard ratio] }\end{array}$} & \multirow[t]{2}{*}{ SE } & \multirow[t]{2}{*}{ Weight } & \multirow[t]{2}{*}{$\begin{array}{l}\text { Hazard ratio IV, } \\
\text { random, } 95 \% \mathrm{Cl}\end{array}$} & \multirow[t]{2}{*}{ Year } & \multicolumn{2}{|c|}{$\begin{array}{l}\text { Hazard ratio IV, } \\
\text { random, } 95 \% \mathrm{CI} \\
\end{array}$} & \\
\hline & & & & & & & & \\
\hline Chromecki & 0.140 & 0.091 & $8.8 \%$ & $1.15[0.96,1.37]$ & 2011 & & & \\
\hline Yafi & 0.693 & 0.302 & $3.8 \%$ & $2.00[1.11,3.61]$ & 2011 & & & \\
\hline Ouzzane & 0.737 & 0.255 & $4.6 \%$ & $2.09[1.27,3.44]$ & 2011 & & & \\
\hline Zhang & 0.683 & 0.163 & $6.8 \%$ & $1.98[1.44,2.73]$ & 2012 & & & \\
\hline Rouprêt & 0.270 & 0.138 & $7.5 \%$ & $1.31[1.00,1.72]$ & 2012 & & & \\
\hline Kim & 0.068 & 0.246 & $4.8 \%$ & $1.07[0.66,1.73]$ & 2015 & & & \\
\hline Shibing & 0.497 & 0.153 & $7.1 \%$ & $1.64[1.22,2.22]$ & 2016 & & & \\
\hline Subtotal $(95 \% \mathrm{Cl})$ & & & $43.3 \%$ & $1.50[1.22,1.84]$ & & & & \\
\hline \multicolumn{8}{|c|}{ Heterogeneity: $\tau^{2}=0.04 ; \chi^{2}=15.56, \mathrm{~d} f=6(p=0.02) ; l^{2}=61 \%$} & \\
\hline \multicolumn{8}{|c|}{ Test for overall effect: $Z=3.88(p=0.0001)$} & \\
\hline \multicolumn{8}{|c|}{ 1.1.2. Only ureter plus dominant lesion } & \\
\hline Raman & 0.207 & 0.180 & $6.3 \%$ & $1.23[0.86,1.75]$ & 2009 & & & \\
\hline Isbarn & 0.030 & 0.116 & $8.1 \%$ & $1.03[0.82,1.29]$ & 2009 & & & \\
\hline Favaretto & 0.262 & 0.304 & $3.8 \%$ & $1.30[0.72,2.36]$ & 2010 & & & \\
\hline Milojevic & 0.262 & 0.378 & $2.8 \%$ & $1.30[0.62,2.73]$ & 2011 & & & \\
\hline Tai & -0.105 & 0.468 & $2.0 \%$ & $0.90[0.36,2.25]$ & 2016 & & & \\
\hline Subtotal $(95 \% \mathrm{Cl})$ & & & $23.0 \%$ & $1.11[0.93,1.32]$ & & & & \\
\hline \multicolumn{8}{|c|}{ Heterogeneity: $\tau^{2}=0.00 ; \chi^{2}=1.38, \mathrm{~d} f=4(p=0.85) ; I^{2}=0 \%$} & \\
\hline \multicolumn{8}{|c|}{ Test for overall effect: $Z=1.13(p=0.26)$} & \\
\hline \multicolumn{9}{|l|}{ 1.1.3. Both location } \\
\hline Ouzzane & 1.089 & 0.265 & $4.4 \%$ & $2.97[1.77,4.99]$ & 2011 & & & \\
\hline Yafi & 1.386 & 0.373 & $2.9 \%$ & $4.00[1.93,8.31]$ & 2011 & & & \\
\hline Rouprêt & 0.231 & 0.118 & $8.0 \%$ & $1.26[1.00,1.59]$ & 2012 & & & \\
\hline Kim & 0.525 & 0.271 & $4.3 \%$ & $1.69[0.99,2.88]$ & 2015 & & & \\
\hline Shibing & 0.735 & 0.163 & $6.8 \%$ & $2.09[1.52,2.87]$ & 2016 & & & \\
\hline Subtotal $(95 \% \mathrm{Cl})$ & & & $26.4 \%$ & $2.07[1.40,3.06]$ & & & & \\
\hline \multicolumn{9}{|c|}{ Heterogeneity: $\tau^{2}=0.14 ; \chi^{2}=17.72, \mathrm{~d} f=4(p=0.001) ; p^{2}=77 \%$} \\
\hline \multicolumn{9}{|c|}{ Test for overall effect: $Z=3.64(p=0.0003)$} \\
\hline \multicolumn{9}{|c|}{ 1.1.4. Only ureter plus both location } \\
\hline Waseda & 0.405 & 0.149 & $7.2 \%$ & $1.50[1.12,2.01]$ & 2015 & & & \\
\hline Subtotal $(95 \% \mathrm{Cl})$ & & & $7.2 \%$ & $1.50[1.12,2.01]$ & & & & \\
\hline \multicolumn{9}{|c|}{ Heterogeneity: not applicable } \\
\hline \multicolumn{9}{|c|}{ Test for overall effect: $Z=2.72(p=0.007)$} \\
\hline Total $(95 \% \mathrm{Cl})$ & & & $100.0 \%$ & $1.52[1.31,1.76]$ & & & & \\
\hline \multicolumn{9}{|c|}{ Heterogeneity: $\tau^{2}=0.06 ; \chi^{2}=47.51, \mathrm{~d} f=17(p=0.0001) ; P^{2}=64 \%$} \\
\hline \multicolumn{6}{|c|}{ Test for overall effect: $Z=5.59(p<0.00001)$} & $0.2 \quad 0.5$ & 1 & 5 \\
\hline \multicolumn{6}{|c|}{ Test for subgroup differences: $\chi^{2}=11.16, \mathrm{~d} f=3(p=0.01), I^{2}=73.1 \%$} & With UI be & Withou & tte \\
\hline
\end{tabular}

Figure 2. Meta-analysis of the effect of ureteral involvement on cancer-specific survival in multivariable analysis 
sised better CSS in patients without UI. Sensitivity analysis by sequential omission of individual studies was performed to assess the stability of the results in subgroups with significant heterogeneity. The analysis indicated that the studies of Chromecki et al. [20] and Rouprêt et al. [22] were the main sources of heterogeneity in the first and third subgroups, respectively.

Two studies compared HRs for CSS among patients with organ-confined disease. The comparison included 2353 patients. The meta-analysis of those studies also emphasised differences in CSS. Among patients with organ-confined diseases, those with $\mathrm{Ul}$ had a worse prognosis $(\mathrm{HR}=1.31$, 95\% Cl: 1.02-1.69; $p=0.035$; Figure 3). Heterogeneity was not observed $\left(I^{2}=0 \%\right)$, and the fixed effects model was used.

Ureteral involvement as a predictor of OS was reported in five studies. The data were not homo- geneous $\left(I^{2}=78 \%\right)$, and the random effects model was used. Cumulative analysis showed that $U I$ is also a significant predictor of OS. The pooled HR for patients with versus those without UI was 1.39 (95\% Cl: $1.11-1.74 ; p=0.004$ ). Funnel plot inspection revealed no indication of publication bias. Multivariable-adjusted HRs for each study are shown in Figure 4.

Furthermore, a subgroup analysis indicated that a difference in OS was only observed in the third subgroup (renal pelvis vs. renal pelvis and ureter). The pooled HR was 1.96 (95\% Cl: 1.58-2.44). Heterogeneity was not observed $\left(R^{2}=0 \%\right)$. When $\mathrm{UI}$ was defined as a tumour located only in the ureter (subgroup 1) or as a tumour located only in the ureter and as a dominant lesion in that location (subgroup 2), no differences in OS were found. Sensitivity analysis indicated that the study of Kim et al. affected the summary result in the first subgroup [24].

$\begin{array}{lccccccc}\text { Study or subgroup } & \begin{array}{c}\text { Log } \\ \text { [hazard ratio] }\end{array} & \text { SE } & \text { Weight } & \begin{array}{c}\text { Hazard ratio IV, } \\ \text { fixed, 95\% Cl }\end{array} & \text { Year } & \text { Hazard ratio } \\ \text { IV, fixed, 95\% Cl }\end{array}$

Figure 3. Meta-analysis of the effect of ureteral involvement on cancer-specific survival in patients with organ-confined upper tract urothelial carcinoma

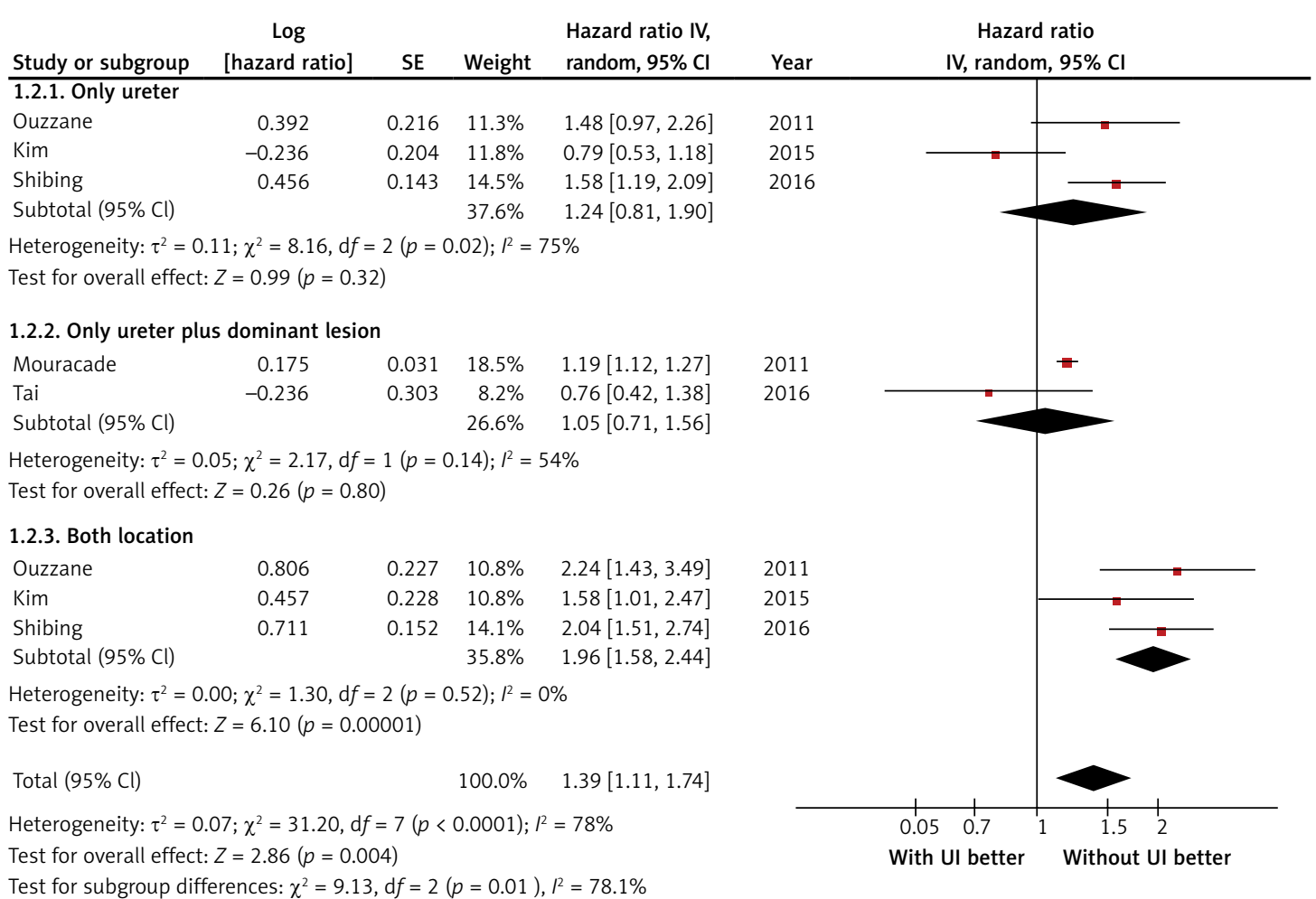

Figure 4. Meta-analysis of the effect of ureteral involvement on overall survival in multivariable analysis 


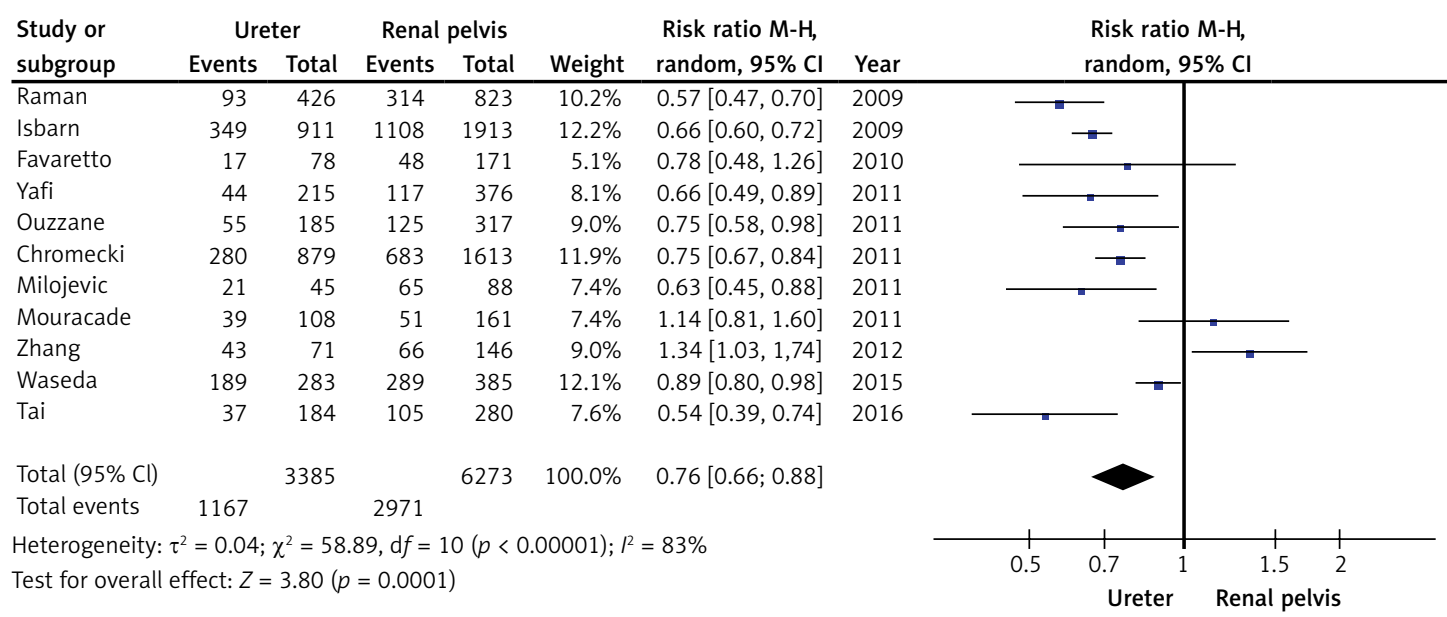

Figure 5. Meta-analysis of tumour location as predictor of pT3-4 tumour stage

Ten studies provided sufficient information to calculate the RR for locally advanced disease (pT3/4) stratified by tumour location (ureter vs. renal pelvis). In those studies, the number of patients who had organ and non-organ-confined diseases were 6272 and 4478, respectively. Statistically significant differences were found in the meta-analysis of tumour location and tumour stage. Patients with a tumour located in the ureter were found to have a lower risk of non-organ-confined disease $(\mathrm{RR}=0.76,95 \% \mathrm{Cl}$ : 0.66-0.88; $p<0.001$; Figure 5). Heterogeneity was significant $\left(R^{2}=83 \%\right)$; sensitivity analysis was conducted to find the source of heterogeneity among all the included studies. The results showed that no single study affected the summary of risk estimates, which indicates that our results are reliable. Visual funnel plot inspection suggested no publication bias.

\section{Discussion}

Our study is the first meta-analysis that attempts to assess the influence of UI, which has different definitions, on prognosis. Previous metaanalyses did not consider patients with ureteral tumours in subgroups. In several studies, tumours located in both the renal pelvis and ureter were classified as multifocal tumours and were not included in the analysis of UI. However, some studies classified patients with multifocal tumours as unifocal based on the site of the dominant lesion. Consequently, identifying the true value of $\mathrm{UI}$ is difficult. The variety of approaches in determining UI limits the use of ureteral tumours as a prognostic factor.

The results of our meta-analysis demonstrated that UI worsens CSS and OS, and analysis of a subset of patients with organ-confined disease revealed that among cases with similar pathologic stages, better CSS was found if the tumour was located in the renal pelvis. Several plausible ex- planations for the aforementioned results exist. First, the anatomical characteristics of the ureter facilitate the spread of tumour cells. The ureter has a thin layer of surrounding adventitia that contains a plexus of blood and lymphatic vessels. Waseda et al. proved that lymphovascular invasion (LVI) is more often observed in ureteral localisation [23], whereas Lee et al. emphasised that $\mathrm{LVI}$ is a practical prognostic parameter, especially in ureteral tumours [27]. Conversely, tumours located in the renal pelvis are surrounded by renal parenchyma and perihilar adipose tissue, which is a natural barrier that is capable of containing the spread of tumour cells, and provides wider surgical margins and enables easier resection. However, some studies showed worsened survival outcomes for pelvis tumours, which could be explained by the thinner muscularis layer in the renal pelvis compared with that in the lower part of the ureter where $70 \%$ of ureteral tumours are located. Nevertheless, these studies were restricted by their small sample sizes [28, 29].

Higher hydronephrosis prevalence in ureteral tumours could also explain the differences in survival. Hydronephrosis is a well-established prognostic parameter for bladder cancer, and the recent meta-analysis by Cao et al. indicated that hydronephrosis is correlated with worsened OS among patients with UTUC [30]. In addition, Morizane et al. showed that preoperative serum creatinine level has an effect on CSS among patients who underwent nephroureterectomy [31]. The influence of hydronephrosis on survival could be mainly explained by the associated multiorgan dysfunction. Impaired renal ability to maintain fluid and electrolyte homeostasis results in accumulation of toxic metabolic products, which subsequently increases the risk of cardiovascular events and deaths from any cause independent of comorbidities [32]. Preoperative hydronephrosis 
in patients with UTUC is also related to more pronounced glomerular filtration rate (GFR) deterioration after RNU [33], thereby seriously restricting the use of cisplatin-based adjuvant chemotherapy [34].

The difference in survival between pelvic and ureteral tumours among patients with organconfined disease was a surprising finding in this meta-analysis. Some authors previously attempted to assess survival in patients with UTUC based on tumour stage and location. Most of the studies reported similar prognosis when ureteral and renal pelvic tumours were matched for tumour stage [35, 36]. However, Kim et al. predicted the development of muscle-invasive bladder cancer (MIBC) after RNU. Their study emphasised that tumour location within the ureter was an independent risk factor for MIBC and that patients with MIBC had a worsened CSS compared with those without MIBC. Additionally, stratification analysis for matching pathologic stage was performed and revealed that MIBC was correlated with significantly poorer CSS only in patients with stage $\mathrm{pTa} / \mathrm{T} 1$ UTUC, while no difference in CSS among patients with tumour stage $\geq \mathrm{pT} 2$ was noted [37].

This current analysis also emphasised some interesting differences in pathologic characteristics based on the tumour location. Locally advanced tumours (pT3/4) were more frequently observed in the pyelocaliceal system, which is in contrast to findings from a previous meta-analysis by Wu et al., who reported no difference in the percentage of pT3/4 lesions between the two locations [10]. This conflicting finding could be attributed to differences in exclusion criteria. In the study of Wu et al., the stage distribution of tumours might have been influenced by the patients who received preoperative treatment and the inclusion of studies with a smaller sample size. Symptomatic obstruction of the urinary tract is frequently observed in patients with ureteral tumours, which could explain the earlier diagnosis and the lower tumour stage.

The subgroup analysis according to the definition of $U \mathrm{Ul}$ revealed a different result in one particular group. The estimated pooled HRs indicated that UI worsened CSS and OS. However, in subgroup 2, where UI was defined as the dominant lesion in the ureter, the analysis found no discrepancy in survival between patients with and those without UI. The higher percentage of more advanced tumours in the renal pelvis suggests that patients with both regional tumours were included in the non-Ul group. Moreover, Waseda et al. suggested that tumours in both locations should be considered as UI independently of tumour stage in the ureter. The author proved that no difference in survival between patients with both regional tumours and patients with tumours located only in the ureter exists [23]. Therefore, patients with tumours in two distinct locations should be taken into account in the analysis of UI instead of the analysis of tumour focality.

This meta-analysis has some limitations. The included studies were mainly retrospective - hence the heterogeneity in the analysis. Some studies possibly failed to identify survival because of relatively short follow-up periods. The included studies were performed in diverse geographical settings (e.g., Europe, Asia, North America), and some authors presented international and multi-institutional results. This geographical diversity might have generated differences in several factors influencing tumour characteristics, including genetic, cultural, and environmental factors [38]. Additionally, our meta-analysis included five studies from Asia, where the population of patients with UTUC is known to differ from that in Western countries and where UTUC is most common in females [39, 40]; transitional cell carcinoma is the most common urological cancer in some Eastern countries [8]. Furthermore, tumours frequently occur in the ureter, and multifocality is not a predictive factor for prognosis [40] Most of the included studies excluded patients with neoadjuvant therapy, and patients received adjuvant therapy in some studies. The analysis also failed to consider other factors that could be a source of heterogeneity and reflected in the results, such as surgical technique, tumour size, or presence of carcinoma in situ.

In conclusion, despite the limitations, the current meta-analysis was able to emphasise worse overall and cancer-specific survival of ureteral vs pelvicalyceal tumours. Our results represent the most robust proof of the difference in the biological potential of ureteral UTUC. We also recommend that researchers should not consider the presence of both regional tumours as unifocal based on the dominant lesion.

\section{Conflict of interest}

The authors declare no conflict of interest.

\section{References}

1. Munoz JJ, Ellison LM. Upper tract urothelial neoplasms: incidence and survival during the last 2 decades. J Urol 2000; 164: 1523-5.

2. Siegel RL, Miller KD, Jemal A. Cancer Statistics, 2017. CA Cancer J Clin 2017; 67: 7-30.

3. Margulis V, Shariat SF, Matin SF, et al. Outcomes of radical nephroureterectomy: a series from the Upper Tract Urothelial Carcinoma Collaboration. Cancer 2009; 115: 1224-33.

4. Roupret M, Babjuk M, Comperat E, et al. European association of urology guidelines on upper urinary tract 
urothelial cell carcinoma: 2017 update. Eur Urol 2018; 73: 111-22.

5. Lughezzani G, Burger M, Margulis V, et al. Prognostic factors in upper urinary tract urothelial carcinomas: a comprehensive review of the current literature. Eur Urol 2012; 62: 100-14.

6. Clements T, Messer JC, Terrell JD, et al. High-grade ureteroscopic biopsy is associated with advanced pathology of upper-tract urothelial carcinoma tumors at definitive surgical resection. J Endourol 2012; 26: 398-402.

7. Park S, Hong B, Kim CS, Ahn H. The impact of tumor location on prognosis of transitional cell carcinoma of the upper urinary tract. J Urol 2004; 171: 621-5.

8. Akdogan B, Dogan HS, Eskicorapci SY, Sahin A, Erkan I, Ozen $\mathrm{H}$. Prognostic significance of bladder tumor history and tumor location in upper tract transitional cell carcinoma. J Urol 2006; 176: 48-52.

9. Petrelli F, Yasser Hussein MI, Vavassori I, Barni S. Prognostic factors of overall survival in upper urinary tract carcinoma: a systematic review and meta-analysis. Urology 2017; 100: 9-15.

10. Wu Y, Dong Q, Liu L, Han P, Wei Q. The impact of tumor location and multifocality on prognosis for patients with upper tract urothelial carcinoma: a meta-analysis. Sci Rep 2014; 4: 6361.

11. Liberati A, Altman DG, Tetzlaff J, et al. The PRISMA statement for reporting systematic reviews and meta-analyses of studies that evaluate health care interventions: explanation and elaboration. J Clin Epidemiol 2009; 62: 1-34.

12. Kwon Y, Lemieux M, McTavish J, Wathen N. Identifying and removing duplicate records from systematic review searches. J Med Libr Assoc 2015; 103: 184-8.

13. Isbarn H, Jeldres C, Shariat SF, et al. Location of the primary tumor is not an independent predictor of cancer specific mortality in patients with upper urinary tract urothelial carcinoma. J Urol 2009; 182: 2177-81.

14. Mouracade P, Velten $M$, Gigante $M$, et al. Factors impacting survival in patients with upper tract urothelial carcinoma undergoing radical nephroureterectomy. Can J Urol 2012; 19: 6105-10.

15. Raman JD, Ng CK, Scherr DS, et al. Impact of tumor location on prognosis for patients with upper tract urothelial carcinoma managed by radical nephroureterectomy. Eur Urol 2010; 57: 1072-9.

16. Favaretto RL, Shariat SF, Chade DC, et al. The effect of tumor location on prognosis in patients treated with radical nephroureterectomy at Memorial Sloan-Kettering Cancer Center. Eur Urol 2010; 58: 574-80.

17. Yafi FA, Novara G, Shariat SF, et al. Impact of tumour location versus multifocality in patients with upper tract urothelial carcinoma treated with nephroureterectomy and bladder cuff excision: a homogeneous series without perioperative chemotherapy. BJU Int 2012; 110: 7-13.

18. Milojevic B, Djokic M, Sipetic-Grujicic S, et al. Upper urinary tract transitional cell carcinoma: location is not correlated with prognosis. BJU Int 2012; 109: 1037-42.

19. Ouzzane A, Colin P, Xylinas E, et al. French Collaborative National Database on UUT-UC. Ureteral and multifocal tumours have worse prognosis than renal pelvic tumours in urothelial carcinoma of the upper urinary tract treated by nephroureterectomy. Eur Urol 2011; 60: 1258-65.

20. Chromecki TF, Cha EK, Fajkovic H, et al. The impact of tumor multifocality on outcomes in patients treated with radical nephroureterectomy. Eur Urol 2012; 61: 245-53.

21. Zhang X, Zhu Z, Zhong S, XU T, Shen Z. Ureteral tumours showing a worse prognosis than renal pelvis tumours may be attributed to ureteral tumours more likely to have hydronephrosis and less likely to have haematuria. World J Urol 2013; 31: 155-60.

22. Rouprêt M, Hupertan V, Seisen T, et al. Prediction of cancer specific survival after radical nephroureterectomy for upper tract urothelial carcinoma: development of an optimized postoperative nomogram using decision curve analysis. J Urol 2013; 189: 1662-9.

23. Waseda Y, Saito K, Ishioka J, et al. Ureteral involvement is associated with poor prognosis in upper urinary tract urothelial carcinoma patients treated by nephroureterectomy: a multicenter database study. Eur Urol Focus 2015; 2: 296-302.

24. Kim HS, Jeong CW, Kwak C, Kim HH, Ku JH. Can body mass index predict survival outcomes in patients treated with radical nephroureterectomy for upper-tract urothelial carcinoma? Int Urol Nephrol 2015; 47: 1311-20.

25. Tai YSS, Chen CHH, Huang CYY, Tai HCC, Wang SMM, $\mathrm{PU}$ YSS. The effect of tumor location on oncologic outcomes in patients with upper urinary tract urothelial carcinoma stratified by pathologic stage. Urol Oncol Orig Investig 2016; 34: 19-25.

26. Shibing Y, Liangren L, Qiang W, et al. Impact of tumour size on prognosis of upper urinary tract urothelial carcinoma after radical nephroureterectomy: a multi-institutional analysis of 795 cases. BJU Int 2016; 118: 902-10.

27. Lee HY, Li CC, Huang CN, et al. Prognostic significance of lymphovascular invasion in upper urinary tract urothelial carcinoma is influenced by tumor location. Ann Surg Oncol 2015; 22: 1392-1400.

28. Van Der Poel HG, Antonini N, Van Tinteren H, Horenblas S. Upper urinary tract cancer: location is correlated with prognosis. Eur Urol 2005; 48: 438-44.

29. Anderstrom C, Johansson SL, Pettersson S, Wahlqvist L. Carcinoma of the ureter: a clinicopathologic study of 49 cases. J Urol 1989; 142: 280-3.

30. Cao J, Zhao X, Zhong Z, Zhang L, Zhu X, XU R. Prognostic value of pre-operative renal insufficiency in urothelial carcinoma: a systematic review and meta-analysis. Sci Rep 2016; 6: 35214.

31. Morizane S, Iwamoto H, Masago T, et al. Preoperative prognostic factors after radical nephroureterectomy in patients with upper urinary tract urothelial carcinoma. Int Urol Nephrol 2013; 45: 99-106.

32. Go AS, Chertow GM, Fan D, McCulloch CE, Hsu C. Chronic kidney disease and the risks of death, cardiovascular events, and hospitalization. N Engl J Med 2004; 351: 1296-305.

33. Hashimoto T, Ohno Y, Nakashima J, et al. Prediction of renal function after nephroureterectomy in patients with upper tract urothelial carcinoma. Jpn J Clin Oncol 2015; 45: 1064-8.

34. Lane BR, Smith AK, Larson BT, et al. Chronic kidney disease after nephroureterectomy for upper tract urothelial carcinoma and implications for the administration of perioperative chemotherapy. Cancer 2010; 116: 2967-73.

35. Hall MC, Womack S, Sagalowsky AI, Carmody T, Erickstad MD, Roehrborn CG. Prognostic factors, recurrence, and survival in transitional cell carcinoma of the upper urinary tract: a 30-year experience in 252 patients. Urology 1998; 52: 594-601.

36. Kim HS, Ku JH, Jeong CW, Kwak C, Kim HH. Laparoscopic radical nephroureterectomy is associated with worse survival outcomes than open radical nephroureterectomy in patients with locally advanced upper tract urothelial carcinoma. World J Urol 2016; 34: 859-69. 
37. Kim KH, You D, Jeong IG, Hong JH, Ahn H, Kim CS. Muscleinvasive bladder cancer developing after nephroureterectomy for upper urinary tract urothelial carcinoma. Urol Oncol 2013; 31: 1643-9.

38. Denis L, Griffiths K, Turkes A. The concept of prevention as a public health strategy for prostate cancer control. Arch Med Sci 2005; 1: 23-33.

39. Chen XP, Xiong GY, Li XS, et al. Predictive factors for worse pathological outcomes of upper tract urothelial carcinoma: experience from a nationwide high-volume centre in China. BJU Int 2013; 112: 917-24.

40. Yang $\mathrm{MH}$, Chen KK, Yen CC, et al. Unusually high incidence of upper urinary tract urothelial carcinoma in Taiwan. Urology 2002; 59: 681-7. 